\title{
Reviewer Acknowledgements 2015
}

\author{
Chris Arme
}

\section{Contributing reviewers}

Parasites \& Vectors would like to thank everyone who reviewed for the journal in 2015.

Rabindra Abeyasinghe

Philippines

Nicole Achee

United States

Mulugeta Aemero

Ethiopia

Yaw Afrane

Ghana

Philip Agnew

France

Lelo Agola

Tanzania

Leopoldina Aguirre Macedo

Mexico

Fola Agusto

United States

Arunee Ahantarig

Thailand

Hirotsugu Aiga

Japan

Amir Akhavan

Iran

Munir Aktas

Turkey

Mohammad Shafiul Alam

Bangladesh

Pedro Alarcón-Elbal

Spain
Pilar Alberdi

Spain

Abebe Alemu

Ethiopia

Kaio Alevi

Brazil

Neal Alexander

United Kingdom

Carlos Eduardo Almeida

Brazil

Hesham Al-Mekhlafi

Malaysia

Lionel Almeras

France

Salman Al-Shami

Saudi Arabia

Adam H. Al-Shamiri

Yemen

Bulent Alten

Turkey

Barry Alto

United States

Cosme Alvarado-Esquivel

Mexico

Cristian Alvarez Rojas

Australia

John Andersen

United States
Martin Andersson

Sweden

Andrey Andrade

Brazil

José Dilermando Andrade Filho Brazil

Valter Andrade-Neto

Brazil

Renato Andreotti

Brazil

Samer Angelone-Alasaad

Spain

Giada Annoscia

Italy

Desiderato Annoscia

Italy

Brendan Ansell

Australia

Christophe Antonio-Nkondjio

Cameroon

Charles Apperson

United States

Jackson Araujo

Brazil

Márcio Araújo

Brazil

Minerva Arce-Fonseca

Mexico

Correspondence: chris.arme@gmail.com

Huxley Building, Keele University, Keele ST5 5BG, UK 
Peter Armbruster

United States

Chris Arme

United Kingdom

Veronica Armijos

United States

Philip Armstrong

United States

Muhammad Ashfaq

Canada

Marek Asman

Poland

Katherine Atkins

United Kingdom

Stephen Atkinson

United States

Carter Atkinson

United States

Márcia Attias

Brazil

Stephen Attwood

China

Denis Augot

France

Andre Bafica

Brazil

Anna Bajer

Poland

Valdir Balbino

Brazil

Dan Baldassarre

United States

Rania Baleela

United States

\section{Allison Bancroft}

United Kingdom

Claudio Bandi

Italy

Gad Baneth

Israel

Jay Bara

United States

Ricardo Andrade Barata

Brazil
Alan Barbour

United States

Marcelo Barcinski

Brazil

Beatrice Barda

Switzerland

Vanessa Bardella

Brazil

Maria Dolores Bargues

Spain

Steve Barker

Australia

Don Barnard

United States

Joel Barratt

Australia

Roberto Barrera

United States

Jean-Mathieu Bart

Spain

Bruno Bassano

Italy

Paul Bates

United Kingdom

Matthew Baylis

United Kingdom

Norbert Becker

Germany

Nigel Beebe

Australia

Mike Begon

United Kingdom

Jerzy Behnke

United Kingdom

Jasminca Behrmann

Germany

John Beier

United States

Jean-Nicolas Beisel

France

Pablo Beldomenico

Argentina

Carlota Belisário

Brazil
Diana Bell

United Kingdom

Silvana Belo

Portugal

John Paul Benante

United States

Marlene Benchimol

Brazil

Daniel Benesh

United States

Kelly Bennett

United Kingdom

Staffan Bensch

Sweden

Robert Bergquist

Sweden

Scott Bernhardt

United States

Rasa Bernotiene

Lithuania

Colin Berry

United Kingdom

Esmael Besufikad

Ethiopia

Martha Betson

United Kingdom

Frederic Beugnet

France

Tapan Bhattacharyya

United Kingdom

Peter Billingsley

United States

Oliver Billker

United Kingdom

Hadush Birhanu

Belgium

Fadi Bittar

France

Samuel Black

United States

William Black IV

United States

Jenefer Blackwell

Australia 
David Blair

Australia

Damer Blake

United Kingdom

Isobel Blake

United Kingdom

Mariana Boadella

Spain

Marlean Boelaert

Netherlands

Mariana Boité

Brazil

Bethany Bolling

United States

Mark Booth

United Kingdom

Michael Boots

United Kingdom

Fred Bordes

France

Robert Bos

Switzerland

Jérémy Bouyer

Senegal

Maha Bouzid

United Kingdom

Dwight Bowman

United States

Leigh Bowman

United Kingdom

Uffe Braae

Denmark

Laura Braden

Canada

Janette Bradley

United Kingdom

Oliver Brady

United Kingdom

Erika Braga

Brazil

Reginaldo Brazil

Brazil

Edward Breitschwerdt

United States
Alberto Bresciani

Italy

Emanuele Brianti

Italy

William Brieger

United States

Jory Brinkerhoff

United States

Andrew Briscoe

United Kingdom

Dustin Brisson

United States

Basil Brooke

South Africa

Simon Brooker

United Kingdom

Peter Brophy

United Kingdom

Robert Brown

United States

Bryan Brown

United States

Kurt Buchmann

Denmark

Amy Buck

United Kingdom

Alicja Buczek

Poland

Lilian Bueno

Brazil

Ruben Bueno-Mari

Spain

Hugo Bugoro

Solomon Islands

Nancy Bunbury

Seychelles

Stewart Burgess

United Kingdom

Alejandro Cabezas-Cruz

France

Jo Cable

United Kingdom

Beatris Cademartori

Brazil
Marie-Christine Cadiergues

France

Claudia Cafarchia

Italy

Francisco Fernando Calvo

Belgium

Mattia Calzolari

Italy

Vitaliano Cama

United States

Mary Cameron

United Kingdom

Corey L. Campbell

United States

Bronwyn Campbell

Australia

Lindsay Campbell

United States

Jorge Cano

United Kingdom

Cinzia Cantacessi

Australia

Jun Cao

China

Gioia Capelli

Italy

Helene Carabin

United States

Ring Carde

United States

Luis Cardoso

Portugal

Ryan Carnegie

United States

Ana Carolina Carneiro

Brazil

Yannick Caron

Belgium

Simon Carpenter

United Kingdom

Hernán Carrasco

Venezuela

Moacir Carretta

Brazil 
Mark Carrington

United Kingdom

Rudi Cassini

Italy

Ricardo Castillo

United States

Socrates Cavalcanti

Brazil

Serena Cavallero

Italy

Giuliano Cecchi
Ethiopia

Neveu Cedric

France

Frank Cezilly

France

Dave Chadee

Trinidad and Tobago

Rachel Chalmers

United Kingdom

Ricky Chan

United States

Kshitij Chandel

India

Kwang Poo Chang

United States

David Chapman

United States

Lloyd Chapman

United Kingdom

Johannes Charlier

Belgium

Farhana Chaudhry

Pakistan

Robert Cheke

United Kingdom

Xiao-Guang Chen

China

Jia-Xu Chen

China

Qijun Chen

China

Daixiong Chen

China
Xiao-Ping Chen

China

Changde Cheng

United States

Guofeng Cheng

China

Neil Chilton

United States

Jan Chirico

Sweden

Bruno Chomel

United States

Valerie Choumet

France

Philippe Christe

Switzerland

Caroline Chua

Malaysia

Tock Chua

Malaysia

Young-Bae Chung

Republic of Korea

David Civitello

United States

Graham Clark

United Kingdom

Theodore Clark

United States

Joel Coats

United States

Maureen Coetzee

South Africa

Luc Coffeng

Netherlands

Gerald Coles

United Kingdom

Doug Colwell

Canada

Gary Conboy

Canada

Jan Conn

United States

Franz Josef Conraths

Germany
Michael Conway

United States

Vincent Corbel

Benin

Anthony Cornel

United States

Sofia Cortes

Portugal

Carlos Costa

Brazil

Jane Costa

Brazil

Jaime Costales

Ecuador

Mark Costello

New Zealand

James Cotton

United Kingdom

Jean T. Coulibaly

France

Jeroen Coumou

Netherlands

Jose Coura

Brazil

Janelle Couret

United States

Robert Cowie

United States

Douglas Craig

Canada

Lee Crainey

United States

Robert Creed

United States

Thomas Cribb

Australia

Giuseppe Cringoli

Italy

Andrea Crisanti

United Kingdom

Dac Crossley

United States

Ron Crump

United Kingdom 
Jing Cui

China

Narcisa Cunha-E-Silva

Brazil

Elisa Cupolillo

Brazil

Douglas Currie

Canada

Rachel Curtis

United States

Cristina Cutillas

Spain

Sally Cutler

United Kingdom

Andre Cutolo

Brazil

Aleksandro Da Silva

Brazil

Ary Gomes Da Silva

Brazil

João Santana da Silva

Brazil

Itabajara da Silva Vaz Jr

Brazil

Fredrick Dahlgren

United States

Jianfeng Dai

China

John Dalton

United Kingdom

Stefano D'Amelio

Italy

Gianluca D'Amico

Romania

Renita Danabalan

Germany

Filipe Dantas-Torres

Brazil

Frédéric Darriet

France

Paban Dash

India

Sunando Datta

India
Arwid Daugschies

Germany

Hans Dautel

Germany

Rebecca Davidson

Norway

Tim Day

United States

Ujjwal De

India

Jose De La Fuente

Spain

Claudio De Liberato

Italy

Wanderley De Souza

Brazil

Alex Debrah

Ghana

Paron Dekumyoy

Thailand

Leen Delang

Belgium

Helene Delatte

France

Vincent Delespaux

Belgium

Alessandra Della Torre

Italy

Paul Denny

United Kingdom

Lindsay Dent

Austria

Jerome Depaquit

France

Peter Deplazes

Switzerland

Marcel Deponte

Germany

Marketa Derdakova

Slovakia

Shaun Dergousoff

Canada

Kebede Deribe

Ethiopia
Nicole Desbois

France

Ranadhir Dey

United States

Sunil Dhiman

India

Angela Di Cesare

Italy

Abdoulaye Diabate

Burkina Faso

Anastasia Diakou

Greece

Diawo Diallo

Senegal

Fernando Dias

Brazil

Carl Dick

United States

Manlio Dicristina

Italy

Muriel Dietrich

Reunion

Colette Dissous

France

Armel Djenontin

Benin

Rosseau Djouaka

Benin

Henryka Długońska

Poland

Gerhard Dobler

Germany

Andrew Dobson

United Kingdom

Stephen Dobson

United States

Roberto Docampo

United States

Michael J. Doenhoff

United Kingdom

Ana Domingos

Spain

Martin Donnelly

United Kingdom 
Pierre Dorny

Belgium

Gilles Dreyfuss

France

Patrick Driguez

Australia

Michael Dryden

United States

Matthew Dryden

United Kingdom

\section{Ai-Fang Du \\ China}

Mariana Duarte

Brazil

Jitender Dubey

United States

Jean Dubremetz

France

Luc Duchateau

Belgium

Giles Duffield

United States

Dagne Duguma

United States

Remy Durand

France

Georg Duscher

Austria

Isabelle Dusfour

France

Eran Dvir

South Africa

Gregory Ebel

United States

Andrea Egizi

United States

M. A. Ehsan

Bangladesh

Rebecca Eisen

United States

Uwem Ekpo

Nigeria

Ayman El-Badry

Egypt
Naglaa El-Lakkany

Egypt

Ivo Elliot

United Kingdom

John Ellis

Australia

Dia-Eldin Elnaiem

United States

Monica Embers

United States

Aidan Emery

United Kingdom

Nancy Endersby

Australia

David Engman

United States

Marina Ereveeva

United States

Kamil Erguler

Cyprus

Koray Ergunay

Turkey

Ozge Erisoz Kasap

Turkey

Roger Eritja

Spain

Nargys Es-Sette

Morocco

Johan Esterhuizen

Uganda

Maria Esteve-Gassent

United States

Agustin Estrada-Peña

Spain

Roger Evans

United Kingdom

Roy Faiman

Israel

Chia-Kwung Fan

Taiwan Republic of China

Fang Fang

China

Rui Fang

China
Chafika Faraj

Morocco

Ary Faraji

United States

Fernanda Faria

Brazil

Rodolfo Andrés Farlora Zapata

Chile

Mark Fast

Canada

Christina Faust

United States

Ronald Fayer

United States

Nina Fefferman

United States

Xin-Gang Feng

China

Yaoyu Feng

China

Tsegaw Fentie

Ethiopia

Heather Ferguson

United Kingdom

Martina Ferraguti

Spain

Marcelo Ferreira

Brazil

Elizabeth Ferrer

Venezuela

Ezio Ferroglio

Italy

Eric Fèvre

United Kingdom

Elisabeth Fichet-Calvet

Germany

Luiz Tadeu Figueiredo

Brazil

Luciana Figueredo

Brazil

Julio V. Figueroa

Mexico

Jordi Figuerola

Spain 
Paul Fine

United Kingdom

Katja Fischer

Australia

Peter Fischer

United States

Durland Fish

United States

\section{Ana Flisser}

Mexico

Adriana Flores

Mexico

Fernando Flores-Pérez

Mexico

Robin Flynn

United Kingdom

Gabor Foldvari

Hungary

Desmond Foley

United States

Cristina Fonseca

Brazil

Dina Fonseca

United States

Dina Fonseca

United States

Albin Fontaine

France

Fabienne Forton

Belgium

Woodridge Foster

United States

Antonio Frangipane Di Regalbono Italy

Mark Freeman

Malaysia

Simone Freitas

Brazil

Michael French

United Kingdom

Roger Frutos

France

Bao-Quan Fu

China
Hans-Peter Fuehrer

Austria

Màrius Fuentes

Spain

Ricardo Fujiwara

Brazil

Jenna Fyfe

United Kingdom

Endalamaw Gadisa

Ethiopia

Gabriella Gaglio

Italy

Francis Gakuya

Kenya

Eunice Galati

Brazil

Fredy Galvis Ovallos

Brazil

Manoj Gambhir

Australia

Eduardo Garcia-Zepeda

Mexico

Mario Garrido

Spain

Lindsey Garver

United States

Katie Gass

United States

Robin Gasser

Australia

Charles Gauci

Australia

Michael Gaunt

United Kingdom

Alessia Gazzonis

Italy

Timothy Geary

Canada

Stefan Geiger

Brazil

Peter Geldhof

Belgium

Claudio Genchi

Italy
Solange Gennari

Brazil

Mónica Germano

Argentina

Dirk Geysen

Belgium

Paul Giacomin

Australia

Annunziata Giangaspero

Italy

Alessio Giannelli

Italy

Gabriella Gibson

United Kingdom

Wendy Gibson

United Kingdom

Nelly Gidaszewski

France

John Gimnig

United States

Michael Ginger

United Kingdom

Yvette Girard

United States

Pedro Giulianini

Italy

Olivier Glaizot

Switzerland

Robert Glaser

United States

Travis Glenn

China

Andea Gloria-Soria

United States

Katey Glunt

United States

Geoffrey Gobert

Australia

Alexander Gofton

Australia

Nick Golding

United Kingdom

Daniel Gomes

Brazil 
Maria Gomes-Solecki

United States

Jorge Gomez-Marin

Colombia

Andres Gomez-Palacio

Colombia

Suênia Gonçalvez

Brazil

\section{Clara González}

Colombia

Camila Gonzalez Rosas

Colombia

Mary Gonzatti

Venezuela

Catherine Gordon

Australia

David Gorla

Argentina

Nicole Gottenker

United States

Anouk Gouvras

United Kingdom

Nicodem James Govella

Tanzania

M Govindarajan

India

Dennis Grab

United States

Daniel Grabner

Germany

Luigi Gradoni

Italy

Thirssa H. Grando

Brazil

Erik Granquist

Norway

Britton Grasperge

United States

Jeremy Gray

Ireland

Marta Grech

Argentina

Ryan Gregory

Canada
Megan Greischar

Canada

Christoph G. Grevelding

Germany

Parwinder Grewal

United States

Christine Griffin

Ireland

Linda Grigoraki

Greece

Mario Grijalva

United States

Jack Grimes

United Kingdom

Alex Grinberg

New Zealand

Edmundo Grisard

Brazil

Maureen Groer

United States

Arthur Gruber

Brazil

Sarah Guagliardo

United States

Gui-Quan Guan

China

Simon Gubbins

United Kingdom

Herbert Guedes

Brazil

Patrick Guerin

Switzerland

Felipe Guhl

Colombia

Favia Guido

Italy

Jacques Guillot

France

Nayana Gunathilaka

Sri Lanka

Filiz Gunay

Turkey

Shivali Gupta

United States
David Gurarie

United States

Rodrigo Gurgel-Goncalves

Brazil

Ricardo Gürtler

Argentina

Helen Guyatt

France

Karen Haag

Brazil

Silvia Haase

Australia

Andrew Haddow

United States

Stephen Hadfield

United Kingdom

Carina Hall

United States

Sascha Hallett

United States

Jo Halliday

United Kingdom

Omar Hamarsheh

Palestinian Territory Occupied

Patrick Hamilton

United Kingdom

Xiu-Min Han

China

Ahmad Hanafi-Bojd

Iran

Kurt Hanevik

Norway

Limb Hapiara

Australia

Ralph Harbach

United Kingdom

David Harrington

Denmark

Laura Harrington

United States

Leslie Harrison

United Kingdom

Bruce Harrison

United States 
Lara Harrup

United Kingdom

Shimon Harrus

Israel

Mo'awia Hassan

Sudan

John Hawdon

United States

Frances Hawkes

United Kingdom

Hadas Hawlena

Israel

Allen Heath

New Zealand

Helena Helmby

United Kingdom

Andrew Hemphill

Switzerland

Anna Henningsson

Sweden

Conny Herholz

Switzerland

Luis Hernandez

United Kingdom

Geoff Hide

United Kingdom

Yousif Himeidan

Sudan

Joseph Hinnebusch

United States

Alexandra Hiscox

Netherlands

Mary Hodges

Kenya

Jane Hodgkinson

United Kingdom

Adnan Hodžić

Austria

Achim Hoerauf

Germany

Johan Höglund

Sweden

Deirdre Hollingsworth

United Kingdom
Deborah Holt

Australia

Sung-Jong Hong

Republic of Korea

David Hoole

United Kingdom

Martin Horn

Czech Republic

Sándor Hornok

Hungary

Gabriele Hrckova

Slovakia

Larry Hribar

United States

Wei Hu

China

Min Hu

China

Victor Hu

United Kingdom

Xuemei Hu

China

Yan Huang

China

Yiau-Min Huang

United States

Si-Yang Huang

China

Yan-Jang Huang

United States

Leon Eklund Hugo

Australia

Jean-Pierre Hugot

France

Sun Huh

Republic of Korea

Nguyen Manh Hung

China

Peter Hunt

Australia

Hilary Hurd

United Kingdom

Ana Hurtado

Spain
Mae Huynh

United States

Athanasios Iatropoulos

Greece

Roberta Iatta

Italy

Adolfo Ibanez-Justicia

Netherlands

Carlos Ibarra-Cerdeña

Mexico

Adriana Ibelli

Brazil

Ralf Ignatius

Germany

Hideo Imamura

Belgium

Juliana Inoue

Brazil

Furhan Iqbal

Pakistan

Seth Irish

United States

Peter Irwin

Australia

Allison Isaacs

United Kingdom

Mats Isaksson

Sweden

Akira Ito

Japan

Al Ivens

United Kingdom

Abdul Jabbar

Australia

Maxime Jacquet

Switzerland

Thomas Jaenson

Sweden

Dragana Jankovic

United States

Cassie Jansen

Australia

Ana Maria Jansen

Brazil 
Fakhri Jeddi

France

Henrik Jensen

Denmark

Aaron Jex

Australia

Wanzhong Jia

China

Anja Joachim

Austria

Magnus Johansson

Sweden

Brian Johnson

Australia

Doug Jones

United States

Malcolm Jones

Australia

Frans Jongejan

Netherlands

Luiz Juliano

Brazil

Tae Sung Jung

Republic of Korea

Silvia Justi

Brazil

Martin Kalbe

Germany

Joshua Kamani

Nigeria

Joseph Kamau

Kenya

Basile Kamgang

France

Ayubo Kampango

Mozambique

Helge Kampen

Germany

Panagiotis Karanis

Germany

Grzegorz Karbowiak

Poland

Mohammad Hossein Karimi

Iran
Egil Karlsbakk

Norway

Hirotomo Kato

Japan

Naoko Kato-Hayashi

Japan

Frank Katzer

United Kingdom

Hitoshi Kawada

Japan

Paul Kaye

United Kingdom

Maria Kazimirova

Slovakia

Jennifer Keiser

Switzerland

Steven Kelly

United Kingdom

Louise Kelly-Hope

United Kingdom

Malcolm Kennedy

United Kingdom

Derek Kennedy

Australia

Ambaye Kenubih

Ethiopia

Fiona Kenyon

United Kingdom

Stella Kepha

Kenya

Naveed Khan

Pakistan

Maxim Khasnatinov

Russian Federation

Nobuhide Kido

Japan

Christian Kiffner

Germany

Gerry Killeen

Tanzania

Dong Min Kim

Taiwan Republic of China

Phillipa King

United Kingdom
Carsten Kirkeby

Denmark

Shinichi Kitamura

Japan

Jovin Kitau

Tanzania

Sawanyawisuth Kittisak

Taiwan Republic of China

Sonia Kjos

United States

Claudia Klein

Canada

Terry Klein

United States

Daniel Kline

United States

Stephen Klotz

United States

Stefanie Knopp

Switzerland

Kevin Kobylinski

Thailand

Alicia Kocisova

Slovakia

Lizette Koekemoer

South Africa

Jacob Koella

France

Constantianus J.M. Koenraadt

Netherlands

Ismail Koltas

Turkey

Nicholas Komar

United States

Yoon Kong

Republic of Korea

Pasi Korhonen

Australia

Aneta Kostadinova

Czech Republic

Linda Kothera

United States

Michalis Kotsyfakis

Czech Republic 
Benjamin Koudou

United Kingdom

Artemis Koukounari

United Kingdom

Moritz Kraemer

United Kingdom

E Krafsur

United States

Lena Krayter

Germany

Michael Kristensen

Denmark

Jürgen Krücken

Germany

Szu-Cheng Kuo

Taiwan Republic of China

Francesco La Torre

Italy

Norma Labarthe

Brazil

Pierrick Labbé

France

Lisa Labbé Sandelin

Sweden

Marcelo Labruna

Brazil

Shannon Ladeau

United States

Thewarach Laha

Thailand

De-Hua Lai

China

Iain Lake

United Kingdom

Patrick Lammie

United States

Sai-Gek Lam-Phua

Singapore

Humberto Lanz-Mendoza

Mexico

Gabriel Laporta

Brazil

Michael Lappin

United States
Abdalla Latif

South Africa

Maria Stefania Latrofa Italy

Audrey Lau

United States

Fabrice Laurent

France

Juan Lauthier

Brazil

Andrea Lawrence

Australia

Scott Lawton

United Kingdom

Phillip Lawyer

United States

Walter Leal

United States

Andy Leisewitz

South Africa

Paul Leisnham

United States

Manigandan Lejeune

Canada

Francisco Lemos

Brazil

Laetitia Lempereur

United Kingdom

Matthias Lendner

Germany

Audrey Lenhart

United States

Samson Leta

Ethiopia

Michael Levin

United States

Michael Levy

United States

Michael Lewis

United Kingdom

Xiangrui Li

China

An-Xing Li

China
Wei Li

China

Youquan Li

China

Guodong Liang

China

Paulo Liedo

United States

Lisette Lieshout

Netherlands

Marshall Lightowlers

Australia

Jiaojiao Lin

China

David Lindsay

United States

Jo Lines

United Kingdom

Yvonne-Marie Linton

United States

Susan Little

United States

Guang-Yuan Liu

China

Guo-Hua Liu

China

Jingze Liu

China

Jun Liu

United States

Jianzhu Liu

China

Mingyuan Liu

China

Quan Liu

China

Xinjian Liu

China

Zhijie Liu

China

Zhi Long Liu

China

Martin Llewellyn

United Kingdom 
David Lloyd

United Kingdom

Mike Lo

United States

Jose Loaiza

Panama

Melissa Lodoen

United States

Carlos Logullo

Brazil

Eric Loker

United States

Fabrizio Lombardo

Italy

Gabriella Lombardo

Italy

Luiz Lopez

Spain

Camila Lorenz

Brazil

Marcelo Lorenzo

Brazil

Vincenzo Lorusso

France

Bertrand Losson

Belgium

Christos Louis

Greece

Van Lun Low

Malaysia

Renke Lühken

Germany

Julius Lukeš

Czech Republic

Zhao-Rong Lun

China

Xue-Nong Luo

China

Zhiyue Lv

China

Issa Lyimo

Tanzania

Andrew Macdonald

United States
Charles Mackenzie

United States

Henry Madsen

Denmark

Mathieu Maheu-Giroux

United Kingdom

Carla Maia

Portugal

Joon Wah Mak

Malaysia

Ben Makepeace

United Kingdom

Joanna Makol

Poland

Arnaldo Maldonado Junior

Brazil

Dave Malone

Tanzania

Sylvie Manguin

France

Am Manonmani

India

Ben Mans

South Africa

Antonio Marco

United Kingdom

Diego Marco

Argentina

Sebastien Marcombe

Lao People's Democratic Republic

Tanguy Marcotty

South Africa

Osvaldo Marinotti

United States

Michele Maroli

Italy

Gisela Marques

Brazil

Josue Martínez-de la Puente

Spain

Sandra Maruyama

Brazil

Santiago Mas-Coma

Spain
Daniel Masiga

Kenya

Bruno Mathieu

France

Vitor Mati

Brazil

Greg Matlashewski

Canada

Petra Matoušková

Czech Republic

Nancy Matowo

Tanzania

Nubia Matta

Colombia

Simonetta Mattiucci Italy

Aaron Maule

United Kingdom

Isabel Mauricio

Portugal

Humphrey Mazigo

Tanzania

Charles Mbogo

Kenya

Moustapha Mbow

Senegal

Nicky McCreesh

United Kingdom

Bradford McGwire United States

Anna McKee

United States

Don McManus

Australia

Carol McNair

United Kingdom

Samantha McNulty

United States

Henry McSorley

United States

Hamish McWilliam

Australia

Jan Mead

United States 
Jansen Medeiros

Brazil

Matthew Medeiros

United States

Graham Medley

United Kingdom

Kim Medley

United States

Jolyon Medlock

United Kingdom

Paula Medone

Argentina

Bastiaan Meerburg

Netherlands

Rudolf Meier

Singapore

Rudy Meiswinkel

Italy

Fábio Melo

Brazil

Sergio Mendonca

Brazil

Vagner Mendonça

Brazil

Pedro Mendoza

Mexico

Carlos Mendoza-Palmero

Mexico

Louisa Messenger

United Kingdom

Antonios Michaelakis

Greece

Janet Midega

Kenya

Eve Miguel

France

Andrei Mihalca

Romania

Jocelyn Millar

United States

Dennis Minchella

United States

Kate Mitchell

United Kingdom
Luke Mitchell

United States

Kalyan Mitra

India

David Modrý

Czech Republic

Chang Moh Seng

Malaysia

Norhayati Moktar

Malaysia

Goudarz Molaei

United States

Alvaro Molina-Cruz

United States

David Molyneux

United Kingdom

Dinesh Mondal

Bangladesh

Paul Monis

Australia

Fernando Monteiro

Brazil

Antonio Montresora

Switzerland

Sarah Moore

United Kingdom

Daryl Moorhead

United States

Jonas Morales Filho

Brazil

Jorge Morales-Montor

Mexico

Serge Morand

France

Alessandra Morassutti

Brazil

František Moravec

Czech Republic

Felipe Moreira

Brazil

Eric Morgan

United Kingdom

Neil Morley

United Kingdom
Renato Mortara

Brazil

Rinaldo Mota

Brazil

Maysa Motoki

Brazil

Karine Mouline

France

Adrian Mountford

United Kingdom

Sara Moutailler

France

Joachim Mueller

Switzerland

Dennis Muhanguzi

Uganda

Simon Muhumuza

Uganda

Grace Mulcahy

Ireland

Andargachew Mulu

Ethiopia

Jason Mulvenna

Australia

Hetron Munang'andu

Norway

Givemore Munhenga

South Africa

Francesc Muñoz-Muñoz

Spain

Cllaudia Munoz-Zanzi

United States

Courtney Murdock

United States

Antonio Muro

Spain

Julie Mustard

United States

Yasen Mutafchiev

Bulgaria

Karuppiah Muthumani

United States

Ephantus J Muturi

United States 
Joseph Mwangangi

Kenya

Clement Mweya

Tanzania

Pauline Mwinzi

Kenya

Elmarie Myburgh

United Kingdom

Peter Myler

United States

Mathios Mylonakis

Greece

Byoung-Kuk Na

Republic of Korea

Johanna Nader

United Kingdom

Behzad Nadjm

Viet Nam

Jun Nakagawa

Japan

Minoru Nakao

Japan

Hira Nakhasi

United States

Mark Nanyingi

Kenya

Farooq Nasar

United States

Julieta Nattero

Argentina

Santiago Nava

Argentina

Ousmane Ndiath

Central African Republic

Daniel Neafsey

United Kingdom

Peter Nejsum

Denmark

Helena Ngowi

Tanzania

Gordon Nichols

United Kingdom

Pin Nie

China
Birgit Nikolay

United Kingdom

Kenneth Nilsson

Sweden

Alasdair Nisbet

United Kingdom

Veeranoot Nissapatorn

Malaysia

Sammy Njenga

Kenya

Karsten Noeckler

Germany

Nivia Nogueira

Brazil

Douglas Norris

United States

Patricia Nuttall

United Kingdom

Philippe Nwane

Cameroon

Anna Obiegala

Germany

Adrián Ochoa-Leyva

Mexico

Eric Ochomo

Kenya

Peter Odermatt

Switzerland

Katie O'Dwyer

New Zealand

Nick Ogden

Canada

Maria Ogrzewalska

Brazil

Johanna Ohm

United States

Oivind Oines

Norway

Fredros Okumu

Tanzania

Gaetano Oliva

Italy

Jader Oliveira

Brazil
Jonathan Oliver

United States

Annette Olsen

Denmark

Peter Olson

United Kingdom

Sheila Ons

Argentina

Marinda Oosthuizen

South Africa

Laor Orshan

Israel

Mohammad Oshaghi

Iran

Hugo Osorio

Portugal

Domenico Otranto

Italy

Johnson Ouma

Tanzania

Ellis Owusu-Dabo

Germany

Richard Oxborough

United Kingdom

Krijn Paaijmans

United States

Daniel Paape

United Kingdom

Kerry Padgett

United States

Soumaïla Pagabeleguem

Burkina Faso

Nitu Pages

Spain

Timothy Paget

United Kingdom

Kathleen Palma

United States

Weiqing Pan

China

Rosario Panadero

Spain

Francisco Panzera

Uruguay 
Barbara Paoletti

Italy

Anna Papa

Greece

Elias Papadopoulos

Greece

Andrea Paparini

Australia

Tony Papenfuss

Australia

Daniel Paris

Thailand

Joong-Ki Park

Republic of Korea

Philippe Parola

France

Marilyn Parsons

United States

Ilaria Pascucci

Italy

Lygia Passos

Brazil

Christophe Paupy

France

Alex Pauvolid-Correa

Brazil

Adolfo Paz

Spain

Gustavo Paz

Brazil

Anna Paziewska

Netherlands

Terry Pearson

Canada

James Pecor

United States

Adrian Pelin

Canada

Cedric Pennetier

Benin

Pamela Pennington

Guatemala

Jose Mauro Peralta

Brazil
Felipe Pereira

Brazil

Piyumali Perera

Australia

Susan Perkins

United States

Stefania Perrucci

Italy

Felipe Arley Pessoa

Brazil

Grasielle Pessoa

Brazil

Jody Peters

Australia

Jennifer Peterson

United States

Andrew Peterson

United States

Dusan Petric

Serbia and Montenegro

Martin Pfeffer

Germany

Romina Piccinali

Argentina

Kim Picozzi

United Kingdom

Andrew Pike

United States

Somchai Pinlaor

Thailand

Adriano Pinter

Brazil

Sébastien Pion

France

Sebastian Pita

Uruguay

Robin Plevin

United Kingdom

Nicolas Pocquet

France

Mikhail Pomaznoy

Russian Federation

Alicia Ponte-Sucre

Venezuela
Fleur Ponton

Australia

Travis Porco

United States

Chuck Porter

United States

Aleksandar Potkonjak

Serbia

Robert Poulin

New Zealand

Jeffrey Powell

United States

Penny Powell

United Kingdom

Ann Powers

United States

Edoardo Pozio

Italy

Narendran Pradeep Kumar

India

David Price

United States

Christian Probst

Brazil

Yiannis Proestos

Cyprus

Zelia Profeta

Brazil

Boonhiang Promdonkoy

Thailand

Jorian Prudhomme

France

Andrea Pugliese

Italy

Rachel Pullan

United Kingdom

Whitney Qualls

United States

Rupert Quinnell

United Kingdom

Martha Quinones

Colombia

Tariq Qureshi

United States 
Juan Antonio Raga

Spain

Rao Ramakrishna

United States

Juan David Ramirez

Colombia

Reda Ramzy

Egypt

Shiwanthi Ranasinghe

Australia

Manoranjan Ranjit

India

Renate Ranka

Latvia

Hilary Ranson

United Kingdom

Didier Raoult

France

Vera Rar

Russian Federation

\section{Alberto Rascon}

United States

Norman Ratcliffe

United Kingdom

Ramesh Ratnappan

United States

Jean Baptiste Rayaisse

Burkina Faso

Paul Ready

United Kingdom

Jose Manuel Rebelo

Brazil

Guy Reeves

Germany

Lisa Reimer

United Kingdom

Bobby Reiner

United States

George Reis

Brazil

William Reisen

United States

Michael Reiskind

United States
Jan Remme

France

Magalie René-Martellet

France

Filiberto Reyes-Villanueva

Mexico

Alexis Ribas

Belgium

José Ribeiro

United States

Stephanie Richards

United States

David Richardson

United Kingdom

Jan Richardus

Netherlands

Stanley Rider Jr.

United States

Michael Riehle

United States

Laura Rinaldi

Italy

Scott Ritchie

Australia

Marta Riutort

United States

Myriam Riyad

Morocco

Annapaola Rizzoli

Italy

Tamalee Roberts

Australia

Derrick Robinson

France

Mark Robinson

United Kingdom

Sara Robledo

Brazil

Maria Robles

Argentina

Kat Rock

United Kingdom

Boris Rodenko

United Kingdom
Eduardo Rodrigues

Brazil

Vasco Rodrigues

France

Mauricio Rodriguez

Brazil

Mario Rodríguez

Mexico

David Roiz

France

Frederico Rojas

United Kingdom

Antonieta Rojas De Arias

Paraguay

David Rollinson

United Kingdom

Patricia Romano

Argentina

Roberto Romi

Italy

Thomas Romig

Germany

Catherine Ronet

Switzerland

André Luiz Roque

Brazil

Patricia Rosa

United States

Joao Rosa

Brazil

Hannah Rose

United Kingdom

Gabriele Rossi

Australia

Luca Rossi

Italy

Xavier Roura

Spain

Mark Rowland

United Kingdom

Syamal Roy

India

Tatiana Rozental

Brazil 


Miguel Rubio Godoy
Mexico
Jonathan Rushton
United Kingdom
Tanya Russell
Australia
Mike Rust
United States
Una Ryan
Australia
Jae-Sook Ryu
Republic of Korea

Mohammad Khalid Saifullah India

Mostafa Saleh

Egypt

Kamila Sales

Brazil

Maria Anice Sallum

Brazil

Oscar Salomon

Argentina

Richard Samuels

Brazil

Abdallah Samy

United States

Ana Sanchez

Canada

Huldah Sang

United Kingdom

Rosemary Sang

Kenya

Maurício Sant'anna

Brazil

Pablo Santo-Orihuela

Argentina

Jeannie Nascimento dos Santos Corrêa

Brazil

Jacenir Santos-Mallet

Brazil

Maria Margarida Santos-Silva

Portugal

Manolis Saridomichelakis

Greece
Mizuki Sasaki

Japan

Sreenivasan Sasidharan

Malaysia

Paul Saunderson

United States

Katherine Sayler

United States

Vera Margarete Scarpassa

Brazil

Bjoern Schaeffner

Brazil

Juliane Schaer

Germany

Guenter Schaub

Germany

Theo Schetters

Netherlands

Gabriele Schoenian

Germany

Thomas Scott

United States

Faiza Sebti

Morocco

Nagila Secundino

Brazil

Otto Seppälä

Switzerland

Aklilu Seyoum

United Kingdom

Renfu Shao

Australia

Paresh Sharma

India

Andrew Shatrov

Russian Federation

Jeffrey Shaw

Brazil

David Shayman

New Zealand

Sergei Shekhovtsov

Russian Federation

Bang Shen

China
Bo Shen

China

Jilong Shen

China

Chien-Ming Shih

Taiwan Republic of China

Andrew Shinn

United Kingdom

Oliver Shune

Zambia

Afzal Siddiqui

United States

Chadwick Sikaala

Tanzania

Maggy Sikulu

Australia

Cornelia Silaghi

Switzerland

Richard Silayo

United Kingdom

Maria Helena Silva-Filha

Brazil

Jose Silveira

Bouvet Island

Ricardo Silvestre

Portugal

Frederic Simard

Burkina Faso

Andrea Šimková

Czech Republic

Gustave Simo

Cameroon

Fernando Simón

Spain

Bob Sinden

United Kingdom

Brajendra Singh

United States

Sarman Singh

India

Om Singh

India

Upinder Singh

United States 
Marianne Sinka

United Kingdom

Steven Sinkins

United Kingdom

Edward Sinski

Poland

Padet Siriyasatien

Thailand

James Skelton

United States

Jan Slapeta

Australia

Hannah Slater

United Kingdom

Nico Smit

South Africa

Jennifer Smith

United States

Joe Smith

United States

Ryan Smith

United States

Thomas Smith

Switzerland

Cairns Smith

United Kingdom

Michael Smout

Australia

Robert Snow

United Kingdom

Daniel Snyder

United States

Daniel Sojka

Czech Republic

Cheikh Sokhna

France

Philippe Solano

Burkina Faso

Laia Solano-Gallego

Spain

Aldo Solari

Chile

Anthony Solomon

United Kingdom
Pradya Somboon

Thailand

Christina Sommerville

United Kingdom

Marcos Sorgine

Brazil

Jani Sormunen

Finland

Manuel Soto

Spain

Eva Spada

Italy

Olivier Sparagano

United Kingdom

Hein Sprong

Netherlands

Chaichontat Sriworarat

Thailand

Claire Standley

United States

Damien Stark

Australia

Lindsay Starkey

United States

Philip Starks

United States

Cathy Steel

United States

Sasa Stefanic

Switzerland

Mario Steindel

Brazil

Sonja Steinke

Germany

Peter Steinmann

Switzerland

Alexandr Stekolnikov

Russian Federation

Nicole Stephenson

United States

Jamie Stevens

United Kingdom

Dietmar Steverding

United Kingdom
Roger Stich

United States

Patricia Stocco

Brazil

Krzysztof Stojecki

Poland

Beatriz Stolf

Brazil

Wilma Stolk

Netherlands

Chris Stone

Switzerland

Michael Strand

United States

Christina Strube

Germany

Snorre Stuen

Norway

Chunlei Su

United States

Carlos Suarez

Brazil

Sarala Subbarao

India

Vikrant Sudan

India

A.B. Sudeep

India

Michael Sukhdeo

United States

Devi Suman

United States

Xun Suo

China

Sinnathamby Surendran

Sri Lanka

Bernd Sures

Germany

Ben Sutherland

Canada

C. Simone Sutherland

Switzerland

Daniel Swale

United States 
Subramanian Swaminathan

India

Zainulabeuddin Syed

United States

Matias Szabo

Brazil

Valentina Tagliapietra

Italy

Herbie Tanowitz

United States

Mark Taylor

United Kingdom

Martin Taylor

United Kingdom

Louis-Albert Tchuem Tchuente

Cameroon

Alan Rodrigues Teixeira Machado

Brazil

Rafael Teixeira-Neto

Brazil

Sam Telford

United States

Lesly Temesvari

United States

Tatiana Teodoro

Brazil

Olle Terenius

Sweden

Luis Ignacio Terrazas

Mexico

Saravanan Thangamani

United States

Silvana Carvalho Thiengo

Brazil

Frédéric Thomas

France

Samuel Thumbi

United Kingdom

Zhancheng Tian

China

Michel Tibayrenc

Thailand

Juan Timi

Argentina
Swati Tiwari

India

Paolo Tizzani

Italy

Rafael Toledo

Spain

Paul Torgerson

Switzerland

Steve Torr

United Kingdom

Eduardo Caio Torres-Santos

Brazil

Baldwyn Torto

Kenya

Pablo Tortosa

Reunion

Philip Toye

Kenya

Rebecca Traub

Australia

Donato Traversa

Italy

Bruno Travi

United States

Omar Triana

Colombia

Frederic Tripet

United Kingdom

Michele Trotta

Italy

Anastasios Tsaousis

United Kingdom

Stephen Kwok-Wing Tsui

China

Michael Turell

United States

Kevin Tyler

United Kingdom

Preethi Udagama

Sri Lanka

Gerrit Uilenberg

France

Amy Ullmann

United States
Saleh Umair

New Zealand

Isik Unlu

United States

Suryanaryana Upadhyayula

India

Joseph Urban

United States

Juerg Utzinger

Switzerland

Gustavo Valbuena

United States

Anayansi Valderrama

Panama

Glyn Vale

Zimbabwe

Maria Adela Valero

Spain

Gustavo Vallejo

Colombia

E.Tellervo Valtonen

Finland

Andrew Van Den Hurk

Australia

Irma Van Die

Netherlands

Jaap J. Van Hellemond

Netherlands

Vida Van Staden

South Africa

Dana Vanlandingham

United States

Amelie Vantaux

Cambodia

Sophie Vanwambeke

Belgium

André Luís Vanzela

Brazil

Anne Vardo-Zalik

United States

Marie Varloud

France

Jefferson Vaughan

United States 
Alex Vaux

United Kingdom

Muriel Vayssier-Taussat

France

Gert Venter

South Africa

Laia Ventura-Garcia

Spain

José Venzal

Uruguay

Jozef Vercruysse

Belgium

Tine Verdonck

Belgium

Guilherme Verocai

United States

Fabrizia Veronesi

Italy

Paula Vieira

Brazil

Elvina Viennet

Australia

Manuela Vilhena

Portugal

Tatjana Vilibic-Cavlek

Croatia

Maxim Vinarski

Russian Federation

Parnpen Viriyavejakul

Thailand

Christopher Vitek

United States

Roberto Vivancos

United Kingdom

Pavel Vlach

Czech Republic

Petr Volf

Czech Republic

Georg Von Samson-Himmelstjerna

Germany

John Vontas

Greece

Maarten Voordouw

Switzerland
Jan Votýpka

Czech Republic

Indra Vythilingam

Malaysia

Andrea Waeschenbach

United Kingdom

Tania Waghorn

New Zealand

Abdul Waheed

United States

Jitra Waikagul

Thailand

Jessica Waite

United States

David Walker

United States

Anthony Walker

United Kingdom

Edward Walker

United States

Richard Wall

United Kingdom

Cathy Walton

United Kingdom

Joao Wanderley

Brazil

Hua Wang

China

Cheng-Fang Wang

China

Chengming Wang

China

Lian Chen Wang

China

Xuefeng Wang

China

Yuan-Zhi Wang

China

Zhong-Quan Wang

China

Rongjun Wang

China

Xiaoyun Wang

United States
Samuel Wanji

Cameroon

Alon Warburg

Israel

Nicola Wardrop

United Kingdom

David Warhurst

United Kingdom

Robert Waterhouse

Switzerland

Norman Waters

United States

Scott Weaver

United States

Bonnie Webster

United Kingdom

David Weetman

United Kingdom

Junfei Wei

United States

Gary Weil

United States

Mylene Weill

France

Susan Welburn

United Kingdom

Beth Wells

United Kingdom

Christopher Whipps

United States

Terry Whitworth

United States

Steve Whyard

Canada

Giovanni Widmer

United States

Stephen Wikel

United States

Tom Wilke

Germany

Richard Wilkerson

United States

Andrew Williams

Germany 
Kim Williamson

United States

R Alan Wilson

United Kingdom

William Wint

United Kingdom

Margareth Wirth

United States

Angelina Wójcik-Fatla

Poland

Zhongdao Wu

China

Xiang-Yun Wu

China

Nigel Wyatt

United Kingdom

Chao-Ming Xia

China

Lihua Xiao

United States

Jiannong Xu

United States

Min-Jun Xu

China

Wenyue Xu

China

Xuenan Xuan

Japan

Anges Yadouleton

Benin

Chao Yan

China

Tetsuya Yanagida

Japan
Ting-Bao Yang

China

Rui Yang

China

Yu-Rong Yang

China

Ana Yatsuda

Brazil

Matthew Yeo

United Kingdom

Serge Yerbanga

Burkina Faso

Melissa Yoshimizu

United States

Hong You

Australia

Neil Young

Australia

Xue-Jie Yu

United States

Antigoni Zacharopoulou

Greece

Stefania Zanet

Italy

Magdalena Zarowiecki

United Kingdom

Elżbieta Żbikowska

Poland

Yifan Zhai

China

Kai Zhang

United States

Qingfeng Zhang

China
Houshuang Zhang

China

Rui Zhang

China

Sufang Zhang

China

Yong-Zhen Zhang

China

Hongwei Zhang

United States

Shaoruo Zhao

China

Guang-Hui Zhao

China

Yadong Zheng

China

Yong-Tang Zheng

China

Elyes Zhioua

Tunisia

Dong-Hui Zhou

China

Xia Zhou

China

Xing-Quan Zhu

China

Liang Zhu

China

Xinping Zhu

China

Dorothee Zielke

Germany

Annetta Zintl

Ireland 